\title{
The Conditions for the Convergence of Power Scaled Matrices and Applications
}

\author{
Xuzhou Chen ${ }^{1}$, Robert E. Hartwig ${ }^{2}$ \\ ${ }^{1}$ Department of Computer Science, Fitchburg State University, Fitchburg, USA \\ ${ }^{2}$ Department of Mathematics, North Carolina State University, Raleigh, USA \\ E-mail: xchen@fitchburgstate.edu, hartwig@math.ncsu.edu \\ Received February 28, 2011; revised May 30, 2011; accepted June 7, 2011
}

\begin{abstract}
For an invertible diagonal matrix $D$, the convergence of the power scaled matrix sequence $D^{-N} A_{N}$ is investigated. As a special case, necessary and sufficient conditions are given for the convergence of $D^{-N} T^{N}$, where $T$ is triangular. These conditions involve both the spectrum as well as the diagraph of the matrix $T$. The results are then used to privide a new proof for the convergence of subspace iteration.
\end{abstract}

Keywords: Convergence, Iterative Method, Triangular Matrix, Gram-Schmidt

\section{Introduction}

The aim of iterative methods both in theory as well as in numerical settings, is to produce a sequence of matrices $A_{0}, A_{1}, \cdots$, that converges to hopefully, something useful. When this sequence diverges, the natural question is how to produce a new converging sequence from this data. One of these convergence producing methods is to diagonally scale the numbers $A_{N}$ and form the sequence $\left\{D_{N} A_{N}\right\}$. Examples of this are numerous, such as the Krylov sequence $\left(x, A x, A^{2} x, \ldots\right)$, which when divergent can be suitably scaled to yield a dominant eigenvector.

The convergence of power scaled iterative methods and more general power scaled Cesaro sums were studied by Chen and Hartwig $[4,6]$. In this paper, we continue our investigation of this iteration and derive a formula for the powers of an upper triangular matrix, and use this to investigate the convergence of the sequence $\left\{D_{n}^{-N} T^{N}\right\}$.

We also investigate the subspace iterations, which has been started by numerous authorss $[1,3,10,11,15]$, and turn our attention to the case of repeated eigenvalues.

The main contributions of this paper are:

-We present the necessary and sufficient conditions for convergence of power scaled triangular matrices $\left\{D_{n}^{-N} T^{N}\right\}$. We prove that these conditions involve both the spectrum as well as the digraph induced by the matrix $T$.

-We apply the the convergence of power scaled triangular matrices with the explicit expression for the G-S factors of $D^{-N} T^{N}$ [3] and present a new proof of the convergence of simultaneous iteration for the case where the eigenvalues of the matrix $A$ satisfy

$$
\begin{gathered}
\left|\lambda_{1}\right| \geq\left|\lambda_{2}\right| \geq \cdots \geq\left|\lambda_{r}\right|>\left|\lambda_{r+1}\right| \geq \cdots \geq\left|\lambda_{n}\right| \\
\text { and }\left|\lambda_{i}\right|=\left|\lambda_{j}\right| \Rightarrow \lambda_{i}=\lambda_{j} .
\end{gathered}
$$

Because of the explicit expression for the GS factors, and the exact convergence results, our discussion is more precise than that given previously $[12,17]$.

One of the needed steps in our investigation is the derivation a formula for the powers of a triangular matrix $T$, which in turn will allow us to analyze the convergence of $D_{T}^{-N} T^{N}$.

Throughout this note all our matrices will be complex and, as always, we shall use $\|\cdot\|$ and $\rho(\cdot)$ to denote the Euclidean norm and spectral radius of $(\cdot)$.

This paper is arranged as follows. As a preliminary result, a formula for the power of an upper triangular matrix is presented in Section 2. It is shown in Section 3 that the convergence of $D_{T}^{-N} T^{N}$ is closely related to the digraph induced by $T$. Section 4 is the main section in which convergence of general power scaled sequence $D^{-N} A_{N}$ is investigated and this, combined with path conditions in Section 3, is then used to discuss the convergence of $D^{-N} T^{N}$. As an application we analyze the convergence results for subspace iterations, in which the eigenvalues are repeated, but satisfy a peripheral constraint. 


\section{Preliminary Results}

We first need a couple of preliminary results.

Lemma 2.1. If $\rho(A)<1$ and $0<\varepsilon_{i}<1$, then

$$
\sum_{k=0}^{N} A^{k} \varepsilon_{k}
$$

converges.

Proof. For $f(z)=\sum_{k=0}^{\infty} \varepsilon_{k} z^{k}$, we have

$$
|f(z)|=\left|\sum_{k=0}^{\infty} \varepsilon_{k} z^{k}\right| \leq \sum_{k=0}^{\infty}|z|^{k}
$$

As the geometric summation on the right-hand side has radius of convergence $1, f(z)$ converges for all $\mathrm{z}$ such that $|z|<1$, which in turn tells us that the radius of convergence of $f(z)$ is no less than 1. Therefore, from Theorem 6.2.8. of [8], $f(A)$ converges.

Next consider the triangular matrix

$$
\boldsymbol{U}=\left[\begin{array}{cccc}
\mu_{1} & u_{12} & \cdots & u_{1 n} \\
0 & \mu_{2} & \ddots & \vdots \\
\vdots & \ddots & \ddots & u_{n-1, n} \\
0 & \cdots & 0 & \mu_{n}
\end{array}\right],
$$

which is used in the following characterization of the powers of a trangular matrix.

Lemma 2.2. Let $\boldsymbol{T}=\left[\begin{array}{ccc}\lambda & a^{T} & \beta \\ 0 & U & c \\ 0 & 0 & v\end{array}\right]$ where $a$ and $c$ are column vectors and suppose that

$$
\boldsymbol{T}^{N}=\left[\begin{array}{ccc}
\lambda^{N} & a_{N}^{T} & \beta_{N} \\
0 & U^{N} & c_{N} \\
0 & 0 & v^{N}
\end{array}\right]
$$

then

$$
\begin{aligned}
\beta_{N}= & \beta\left(\sum_{k=0}^{N-1} \lambda^{N-k-1} v^{k}\right) \\
& +\sum_{k=0}^{N-2}\left(a^{T} U^{k} c\right)\left(\sum_{i=0}^{N-k-2} \lambda^{N-k-i-2} v^{i}\right)
\end{aligned} .
$$

in particular,

1) if $\lambda=0$, then

$$
\beta_{N}=\beta v^{N-1}+\sum_{k=0}^{N-2}\left(a^{T} U^{k} c\right) v^{N-k-2},
$$

2) if $v=0$, then

$$
\beta_{N}=\beta \lambda^{N-1}+\sum_{k=0}^{N-2}\left(a^{T} U^{k} c\right) \lambda^{N-k-2},
$$

3) if $\lambda \neq 0$ and $\lambda \neq v$, then

$$
\begin{aligned}
\beta_{N}= & \lambda^{N} \beta\left(\frac{1-(v / \lambda)^{N}}{\lambda-v}\right) \\
& +\lambda^{N-1} \sum_{k=0}^{N-2} a^{T}\left(\frac{U}{\lambda}\right)^{k} c\left(\frac{1-(v / \lambda)^{N-k-1}}{\lambda-v}\right),
\end{aligned}
$$

4) if $\lambda \neq 0$ and $\lambda=v$, then

$$
\beta_{N}=N \beta \lambda^{N-1}+\lambda^{N-2} \sum_{k=0}^{N-2} a^{T}\left(\frac{U}{\lambda}\right)^{k} c(N-k-1) \text { and (8) }
$$

5) if $\lambda=v=0$, then

$$
\beta_{N}=a^{T} U^{N-2} c .
$$

Proof. It is easily verified by induction that $\boldsymbol{T}^{N}=$ $\left[\begin{array}{cc}T_{1}^{N} & y_{N} \\ O & v^{N}\end{array}\right]$, where

$$
\boldsymbol{T}_{\mathbf{1}}^{\boldsymbol{k}}=\left[\begin{array}{cc}
\lambda & a^{T} \\
O & U
\end{array}\right]^{k}=\left[\begin{array}{cc}
\lambda^{k} & \sum_{j=0}^{k-1} \lambda^{k-j-1} a^{T} U^{j} \\
O & U^{k}
\end{array}\right]
$$

and

$$
\boldsymbol{y}_{N}=\left[\begin{array}{l}
\beta_{N} \\
C_{N}
\end{array}\right]=\sum_{k=0}^{N-1} T_{1}^{N-k-1}\left[\begin{array}{l}
\beta \\
C
\end{array}\right] v^{k}
$$

Now

$$
\begin{aligned}
\boldsymbol{y}_{N} & =\sum_{k=0}^{N-1}\left[\begin{array}{cc}
\lambda^{N-k-1} & \sum_{j=0}^{N-k-2} \lambda^{N-k-j-2} a^{T} U^{k} \\
O & U^{N-k-1}
\end{array}\right]\left[\begin{array}{l}
\beta \\
c
\end{array}\right] v^{k} \\
& =\sum_{k=0}^{N-1}\left[\begin{array}{c}
\beta \lambda^{N-k-1}+\sum_{j=0}^{N-k-2} \lambda^{N-k-j-2} a^{T} U^{k} C \\
U^{N-k-1} c
\end{array}\right] v^{k} \\
& =\left[\begin{array}{c}
\beta \sum_{k=0}^{N-1} \lambda^{N-k-1} v^{k}+\sum_{k=0}^{N-2 N-k-2} \sum_{j=0}^{N-k} \lambda^{N-j-2} a^{T} U^{k} c v^{k} \\
\sum_{k=0}^{N-1} U^{N-k-1} c v^{k}
\end{array}\right]
\end{aligned}
$$

Hence

$$
\begin{aligned}
\beta_{N} & =\beta\left(\sum_{k=0}^{N-1} \lambda^{N-k-1} v^{k}\right)+\sum_{k=0}^{N-2} \sum_{j=0}^{N-k-2} \lambda^{N-k-j-2} a^{T} U^{k} c v^{k} \\
& =\beta\left(\sum_{k=0}^{N-1} \lambda^{N-k-1} v^{k}\right)+\sum_{k=0}^{N-2}\left(a^{T} U^{k} c\right)\left(\sum_{j=0}^{N-k-2} \lambda^{N-k-j-2} v^{k}\right), \\
& =\beta\left(\sum_{k=0}^{N-1} \lambda^{N-k-1} v^{k}\right)+\sum_{j=0}^{N-2}\left(a^{T} U^{j} c\right)\left(\sum_{k=0}^{N-j-2} \lambda^{N-k-j-2} v^{k}\right)
\end{aligned}
$$

completing the proof of (4). The special cases (1) - (5) are easy consequences of (4).

Let us now illustrate how the power of $T$ are related to its digraph. 


\section{The Digraph of $T$}

Suppose $\quad \boldsymbol{T}=\left[\begin{array}{ccc}\lambda & a^{T} & \beta \\ O & U & c \\ 0 & O & v\end{array}\right] \quad$ is an $(n+2) \times(n+2)$

upper triangular matrix. Correspondingly we select $n+2$ nodes $S_{0}, S_{1}, \cdots, S_{n}, S_{n+1}$, and consider the assignment

$$
\begin{array}{lcccccc} 
& S_{0} & S_{1} & \cdots & \cdots & S_{n} & S_{n+1} \\
S_{0} & {\left[\begin{array}{ccccccc}
\lambda & & & a^{T} & & \beta \\
S_{1} & & \mu_{1} & u_{12} & \cdots & u_{1 n} & \\
\vdots & 0 & \mu_{2} & & \vdots & \\
\vdots & O & \ddots & \ddots & u_{n-1, n} & c \\
S_{n} & \vdots & \vdots & \cdots & 0 & \mu_{n} & \\
S_{n+1} & & 0 & \cdots & & v
\end{array}\right]}
\end{array}
$$

with $a=\left[a_{1}, a_{2}, \cdots, a_{n}\right]^{T}$ and $c=\left[c_{1}, c_{2}, \cdots, c_{n}\right]^{T}$.

We next introduce the digraph induced by $T$, i.e. $G=(V, E)$ where $V=\left\{S_{0}, S_{1}, \cdots, S_{n+1}\right\}$ is the vertex set and $E=\left\{\left(S_{i}, S_{j}\right) \mid t_{i j} \neq 0\right\}$ is the edge set. As usual we say $\left(S_{i}, S_{j}\right) \in E$ if and only if $t_{i j} \neq 0$. A path from $S_{j}$ to $S_{k}$ in $G$ is a sequence of vertices $S_{j}=S_{r_{1}}$, $S_{r_{2}}, \cdots, S_{r_{l}}=S_{k}$ with $\left(S_{r_{i}}, S_{r_{i+1}}\right) \in E$, for $i=1, \cdots, l-1$, for some $l$. If there is a path from $S_{j}$ to $S_{k}$, we say that $S_{j}$ has access to $S_{k}$ and $S_{k}$ can be reached from $S_{j}$. We write

$$
\begin{array}{ll}
S_{i} \rightarrow S_{j} & \text { if }\left(S_{i}, S_{j}\right) \in E, \\
S_{i} \rightarrow S_{j} & \text { if there is a path from } S_{i} \text { to } S_{j}, \\
S_{i} \leftarrow \leftrightarrow \rightarrow S_{j} & \text { if } S_{i} \rightarrow \rightarrow S_{j} \text { and } S_{j} \rightarrow \rightarrow S_{i}
\end{array}
$$

Let $\pi=<S_{0}, S_{n+1}>=\left\{S_{p_{1}}, \cdots, S_{p_{t}}\right\}$ be the sandwich set of $S_{0}$ and $S_{n+1}$, i.e., $\left\{S_{p_{1}}, \cdots, S_{p_{t}}\right\}$ is the set of all the nodes from $\left\{S_{1}, \cdots, S_{n}\right\}$ such that $S_{0} \rightarrow \rightarrow S_{p_{i}} \rightarrow \rightarrow$ $S_{n+1}$, i.e., $S_{p_{i}}$ can be reached from $S_{0}$ and has access to $S_{n+1}$. Let us now introduce the notation

$$
\begin{aligned}
a & =\left[a_{p_{1}}, \cdots, a_{p_{t}}\right]^{T}, \\
\hat{U} & =U\left(\begin{array}{l}
p_{1} \cdots p_{t} \\
p_{1} \cdots p_{t}
\end{array}\right), \\
c & =\left[c_{p_{1}}, \cdots, c_{p_{t}}\right]^{T} .
\end{aligned}
$$

Then we have the following result.

Lemma 3.1. $a^{T} U_{C}=a^{T} \hat{U}_{C}$.

Proof. If $a_{i} u_{i j} c_{j} \neq 0$, then $\left(S_{0}, S_{i}\right),\left(S_{i}, S_{j}\right),\left(S_{j}\right.$, $\left.S_{n+1}\right) \in E$, thus $S_{i}, S_{j} \in \pi$, which implies that

$$
a^{T} U c=\sum_{i=1}^{n} \sum_{j=1}^{n} a_{i} u_{i j} c_{j}=\sum_{i \in \pi} \sum_{j \in \pi} a_{i} u_{i j} c_{j} .
$$

This completes the proof. $\square$

This following corollaries are the direct consequences of the above lemma.
Corollary 3.2. If $S_{0} \rightarrow \rightarrow S_{n+1}$ and there is no intermediate node that lies in $\left\{S_{1}, \cdots, S_{n}\right\}$ on any path from $S_{0}$ to $S_{n+1}$, then

1) $S_{0} \rightarrow S_{n+1}$, i.e. $\beta \neq 0$,

2) $a^{T} U^{i} c=a^{T} \hat{U}^{i} C=0$

Corollary 3.3. $a^{T} U^{i} c=a^{T} \hat{U}^{i} c$, for $i=1,2, \cdots$.

We now turn to the main theorem of this section.

Theorem 3.4. Let $\boldsymbol{T}=\left[\begin{array}{cccc}\lambda_{1} & t_{12} & \cdots & t_{1 n} \\ 0 & \ddots & \ddots & \vdots \\ \vdots & & \ddots & t_{n-1, n} \\ 0 & \cdots & 0 & \lambda_{n}\end{array}\right]$ be nonsingular and $\boldsymbol{D}_{T}=\operatorname{diag}(T)=\operatorname{diag}\left(\lambda_{1}, \ldots, \lambda_{n}\right)$. Then, the following statement are equivalent

1) $D_{T}^{-N} T^{N}$ converges.

2) if $S_{i} \rightarrow \rightarrow S_{j}$, then $\left|\lambda_{i}\right|>\left|\lambda_{j}\right|$, i.e. if there is a path from $S_{i}$ to $S_{j}$, then $\left|\lambda_{i}\right|>\left|\lambda_{j}\right|$.

Proof. We prove the theorem by induction on $n$. For $n=2$, $\boldsymbol{T}=\left[\begin{array}{ll}\lambda_{1} & \beta \\ 0 & \lambda_{2}\end{array}\right] \quad$ and $\quad \boldsymbol{M}_{2}^{(N)}=\boldsymbol{D}_{T}^{-N} \boldsymbol{T}^{N}=\left[\begin{array}{cc}1 & \beta_{N} / \lambda_{1}^{N} \\ 0 & 1\end{array}\right]$ where

$$
\frac{\beta_{N}}{\lambda_{1}^{N}}=\left\{\begin{array}{ll}
\beta\left[1-\left(\lambda_{2} / \lambda_{1}\right)^{N}\right] /\left(\lambda_{1}-\lambda_{2}\right) & \text { if } \lambda_{1} \neq \lambda_{2} \\
\beta \cdot N / \lambda_{1} & \text { if } \lambda_{1}=\lambda_{2}
\end{array} .\right.
$$

It is easily seen that the convergence of $M^{(2)}$ implies that of $\frac{\beta_{N}}{\lambda_{1}^{N}}$. Hence if $\lambda_{1}=\lambda_{2}$, then $\beta=0$. Conversely, if $\beta \neq 0$, then $\left|\lambda_{2} / \lambda_{1}\right|<1$ which implies that $M^{(2)}$ converges.

Next, assume that the result holds for all triangular matrices of size $n+1$ or less. Let $T$ be defined as in (12) and set $\boldsymbol{D}_{T}:=\operatorname{diag}\left(\lambda, \mu_{1}, \ldots, \mu_{n}, v\right)=\operatorname{diag}(\lambda, \Delta$, $v)$ which is nonsingular. Consider the vertex set $V=\left\{S_{0}, \cdots, S_{n+1}\right\}$ and the assignment

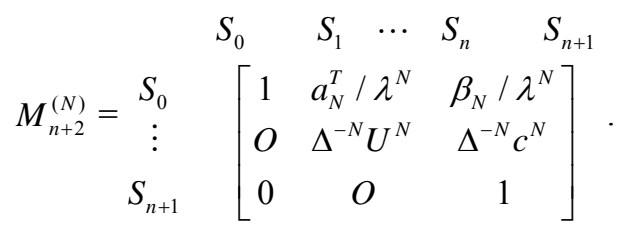

$1) \Rightarrow 2)$. Assume that $M_{n+2}^{(N)}$ converges. Then by induction, both $\left[\begin{array}{cc}\lambda & a^{T} \\ O & U\end{array}\right]$ and $\left[\begin{array}{cc}U & c \\ O & v\end{array}\right]$ obey the theorem. Suppose $S_{i} \rightarrow \rightarrow S_{j}$ in $V$. If $|i-j|<n+1$ we are done since then both endpoints lie in $\left\{S_{0}, \cdots, S_{n}\right\}$ or $\left\{S_{1}, \cdots, S_{n+1}\right\}$. So we only need to consider the case where $S_{i}=S_{0}$ and $S_{j}=S_{n+1}$, i.e. $S_{0} \rightarrow \rightarrow S_{n+1}$.

Subcase (a): There is an intermediate node from 
$\left\{S_{1}, \cdots, S_{n}\right\}$, say $\quad S_{0} \rightarrow \rightarrow S_{p} \rightarrow \rightarrow S_{n+1} \quad(1 \leq p \leq n)$. Then by the induction hypothesis $|\lambda|>\left|\mu_{p}\right|>|v|$, and we are done.

Subcase (b): There is no intermediate node between $S_{0}$ and $S_{n+1}$. In this case $S_{0} \rightarrow S_{n+1}$, and by Corollary 3.2., $\beta \neq 0$ and $a^{T} U^{i} c=a^{T} \hat{U}^{i} c=0$ for arbitrary $i$. Since the sandwich set $\pi$ is empty, we see from Lemma 2.2., that

$$
\lambda^{-N} \cdot \beta_{N}=\left\{\begin{array}{ll}
\beta\left[1-(v / \lambda)^{N}\right] /(\lambda-v) & \text { if } \lambda \neq v \\
\beta \cdot N / \lambda & \text { if } \lambda=v
\end{array} .\right.
$$

Now because we are given that $\lambda^{-N} \beta_{N}$ converges and $\beta \neq 0$, we must have $|v / \lambda|<1$.

Conversely, assume that $S_{i} \rightarrow \rightarrow S_{j} \Rightarrow\left|\lambda_{i}\right|>\left|\lambda_{j}\right|$ and assume that the hypothesis holds for matrices of size $n+1$ or less. Since the graph condition also hold for $\left\{S_{0}, \cdots, S_{n}\right\}$ and $\left\{S_{1}, \cdots, S_{n+1}\right\}$, it follows by the hypothesis that all the entries in $M_{n+2}^{(N)}$ converges, with the possible exception of $\beta_{N} / \lambda^{N}$. Consequently, all we have to show is that $\lambda^{-N} \beta_{N}$ also converges, given the path conditions. Consider

$$
\lambda^{-N} \beta_{N}=\left\{\begin{array}{c}
\beta \frac{1-(v / \lambda)^{N}}{\lambda-v}+ \\
\frac{1}{\lambda} \sum_{i=0}^{N-2} a^{T}\left(\frac{U}{\lambda}\right)^{i} c\left(\frac{1-(v / \lambda)^{N-i-1}}{\lambda-v}\right) \\
\text { if } \lambda \neq v \\
\beta N / \lambda+\frac{1}{\lambda^{2}} \sum_{i=0}^{N-2} a^{T}\left(\frac{U}{\lambda}\right)^{i} c(N-i-1) \\
\text { if } \lambda=v
\end{array}\right.
$$

If $S_{0} \nrightarrow \rightarrow S_{n+1}$, then $S_{0} \rightarrow S_{n+1}$ and therefore $\beta=$ 0 . Moreover, $\pi$ is empty and the right hand side of (15) is zero, i.e. $\lambda^{-N} \beta_{N}=0$ and we are done. So suppose $S_{0} \rightarrow \rightarrow S_{n+1}$ and thus $|\lambda|>|v|$. In this case

$\beta \frac{1-(v / \lambda)^{N}}{\lambda-v}$ converges (possibly to 0 when $\beta=0$ ). Now if $\pi=\varnothing$ then the second term of (15) vanishes by Lemma 2.2. Lastly suppose $\pi \neq \varnothing$, i.e. there are intermediate nodes $S_{p_{1}}, \cdots, S_{p_{t}}$. From Lemma 2.2., we recall that $a^{T} U^{i} C=a^{T} \hat{U}^{i} C$ where

$$
\hat{\boldsymbol{U}}=\left[\begin{array}{lll}
\mu_{p_{1}} & & * \\
& \ddots & \\
O & & \mu_{p_{t}}
\end{array}\right] \begin{aligned}
& S_{p_{1}} \\
& \vdots \\
& S_{p_{t}}
\end{aligned} .
$$

Since for each $i, S_{0} \rightarrow \rightarrow S_{p_{i}} \rightarrow \rightarrow S_{n+1}$, we know that $|\lambda|>\left|\mu_{p_{i}}\right|>|v|$ and thus $|\lambda|>\rho(\hat{U})$. Hence $\rho$ $(\hat{U} / \lambda)<1$ which implies that

$$
a^{T} \sum_{i=0}^{N-2}\left(\frac{\hat{U}}{\lambda}\right)^{i} c \rightarrow a^{T}\left(I-\frac{\hat{U}}{\lambda}\right)^{-1} c .
$$

To complete the proof we observe that

$$
\sum_{i=0}^{N-2}\left(\frac{\hat{U}}{\lambda}\right)^{i}\left(\frac{v}{\lambda}\right)^{N-i-1}
$$

also converges because of Lemma 2.1. with $A=\hat{U} / \lambda$ and $\varepsilon_{i}=(v / \lambda)^{N-i}$.

We at once have, as seen in [3].

Corollary 3.5. Let $T$ be an upper triangular matrix and $\boldsymbol{D}_{T}=\operatorname{diag}(T)=\operatorname{diag}\left(\lambda_{1}, \ldots, \lambda_{n}\right)$. If

$$
\left|\lambda_{1}\right|>\left|\lambda_{2}\right|>\cdots>\left|\lambda_{n}\right|
$$

then $D_{T}^{-N} T^{N}$ converges to an upper triangular matrix of diagonal 1.

We now turn to the main result in this paper. Our aim is to characterize the convergence of $D^{-N} A_{N}$ in terms of the GS factorization of $A_{N}$.

\section{Main Theorem}

Let us denote the set of increasing sequences of $p$ elements taken from $(1,2, \cdots, m)$ by

$$
Q_{p, m}=\left\{I=\left(i_{1}, \cdots, i_{p}\right) \mid 1 \leq i_{1}<\cdots<i_{p} \leq m\right\}
$$

and assume this set $Q_{p, m}$ is ordered lexicographically. Suppose $\langle s: t\rangle:=(s, s+1, \ldots, t)$ is a subsequence of $(1$, $2, \ldots, m)$ and we define

$$
Q_{p}\langle s: t\rangle=\left\{U=\left(u_{1}, \cdots, u_{p}\right) \mid s<u_{1}<\cdots<u_{p}<t\right\} .
$$

Clearly, $Q_{p, m}=Q_{p}\langle 1: m\rangle$.

Suppose $B$ is $m \times n$ matrix of rank $r$. The determinant of a $p \times p$ submatrix of $A \quad(1 \leq p \leq \min$ $(m, n))$, obtained from $A$ by striking out $m-p$ rows and $n-p$ columns, is called a minor of order $p$ of $A$. If the rows and columns retained are given by subscripts (see Householder [9]) $I=\left(i_{1}, \cdots, i_{p}\right) \in Q_{p, m}$ and $J=\left(j_{1}, \cdots, j_{p}\right) \in Q_{p, n} \quad$ respectively, then the corresponding $p \times p$ submatrix and minor are respectively denoted by $A_{J}^{I}$ and $\operatorname{det}\left(A_{J}^{I}\right)$.

The minors for which $I=J$ are called the principal minors of $A$ of order $p$, and the minors with $I=J=(1,2, \cdots, p)$ are referred to as the leading principal minors of $A$.

Let $I=\left(i_{1}, \cdots, i_{p}\right) \in Q_{p, m}$ and $J=\left(j_{1}, \cdots, j_{q}\right) \in Q_{q, m}$. For convenience, we denote by $I\left[i_{k}\right] \in Q_{p-1, m}$ the sequences of $p-1$ elements obtained by striking out the $k$ th element $i_{k}$; while $I(j)$ denotes the sequences of $p+1$ elements obtained by adding a new element $j$ after $i_{k}$, i.e., $I(j)=\left(i_{1}, \cdots, i_{k}, j\right)$. Note that if $i_{p}>j$, then $I(j)$ is not an element of $Q_{p+1, m}$ because it is no longer an increasing sequence. If $p+q \leq m$, we denote the concaternation $\left(i_{1}, \cdots, i_{p}, j_{1}\right.$, 
$\cdots, j_{q}$ ) of $I$ and $J$ by $I J$. It has $p+q$ elements. Again, IJ may not be an element of $Q_{p+q, m}$.

Since the natural sequence $(1,2, \cdots, p)$ of $p$ elements will be used frequently, we particularly denote this sequence by $\langle p\rangle=(1,2, \cdots, p)$; while $\langle p\rangle[t]=(1$, $\cdots, t-1, t+1, \cdots, p)$ is simply denoted by $\langle p \backslash t\rangle$.

Next recall [2] that the volume $\operatorname{Vol}(B)$ of a real matrix $B$, is defined as the product of all the nonzero singular values of $B$. It is known [2] that

$$
\operatorname{Vol}(B)=\sqrt{\sum\left|\operatorname{det}\left(B_{J}^{I}\right)\right|^{2}},
$$

where $B_{J}^{I}$ are all $r \times r$ submatrices of $B$. In particular, if $B$ has full column rank, then

$$
\operatorname{Vol}(B)=\sqrt{\operatorname{det}\left(B^{*} B\right)} .
$$

Lastly, suppose $A=\left[a_{1}, a_{2}, \cdots, a_{r}\right]$ is an $n \times r$ matrix of full column rank and

$$
A=Y G
$$

is its GS factorization so that the columns of $Y=\left[y_{1}\right.$, $\left.y_{2}, \cdots, y_{r}\right]$ are orthogonal and $G$ is $r \times r$ upper triangular matrix of diagonal 1. For $k \leq r$, we define $A_{k}=\left[a_{1}, \cdots, a_{k}\right]$ and

$$
V_{k}=\operatorname{Vol}\left(A_{k}\right) .
$$

It follows directly that

$$
V_{k}=\sqrt{\sum_{I \in Q_{k, m}}\left|\operatorname{det}\left(A_{\langle k\rangle}^{I}\right)\right|^{2}}=\sqrt{\operatorname{det}\left(A_{k}^{*} A_{k}\right)} .
$$

Theorem 4.1. Let $A$ be an $n \times r$ matrix of rank $r$ and let $A=Y G$ be its GS factorization. Then

$$
y_{k l}=\sum_{I \in Q_{l-1, n}} \operatorname{det}\left(A_{\langle l\rangle}^{I(k)}\right) \cdot \overline{\operatorname{det}\left(A_{\langle l-1\rangle}^{I}\right)} / V_{l-1}^{2}
$$

and

$$
g_{j k}=\frac{\operatorname{det}\left(A^{*} A\right)_{\langle j-1\rangle(k)}^{\langle j\rangle} .}{V_{j}^{2}} .
$$

Proof. The result of (22) follows from Theorem 2.1. in [3], while on account of Corollary 2.1. in [3], $G=\left(Y^{*}\right.$ $Y)^{-1} Y^{*} A$. Hence we arrive at

$$
\begin{aligned}
g_{j k} & =\frac{V_{j-1}^{2}}{V_{j}^{2}} \cdot y_{j}^{*} a_{k}=\frac{V_{j-1}^{2}}{V_{j}^{2}} \sum_{l=1}^{n} \overline{y_{l j}} a_{l k} \\
& =\sum_{l=1}^{n} \sum_{t=1}^{j}(-1)^{j+t} \overline{a_{l t}} a_{l k} \operatorname{det}\left(A^{*} A\right)_{\langle j-1\rangle}^{\langle j \backslash t\rangle} / V_{j}^{2}
\end{aligned} .
$$

Because $\sum_{l=1}^{n} \overline{a_{l t}} a_{l k}$ is just the $(t, k)$ element of matrix $A^{*} A$, we see that

$$
g_{j k}=\sum_{t=1}^{j}(-1)^{j+t}\left(\sum_{l=1}^{n} \overline{a_{l t}} a_{l k}\right) \operatorname{det}\left(A^{*} A\right)_{\langle j-1\rangle}^{\langle j \backslash t\rangle} / V_{j}^{2},
$$

which is the Laplace expansion along column $j$ of $\operatorname{det}\left(A^{*} A\right)_{\langle j-1\rangle(k)}^{\langle j\rangle}$. Thus

$$
g_{j k}=\frac{\operatorname{det}\left(A^{*} A\right)_{\langle j-1\rangle(k)}^{\langle j\rangle}}{V_{j}^{2}}
$$

completing the proof.

Remark: A different proof of (23) was given in [9, $\mathcal{S}$ 1.4].

For a diagonal matrix $\boldsymbol{D}=\operatorname{diag}\left(d_{1}, \ldots, d_{n}\right)$, we say that $D$ is decreasing, if

$$
\left|d_{1}\right| \geq \cdots \geq\left|d_{n}\right|
$$

Moreover, $D$ is called locally primitive, if it is decreasing and

$$
d_{i}|=| d_{j} \mid \Rightarrow d_{i}=d_{j} .
$$

It is obvious that we can partition a decreasing matrix $D$ as

$\boldsymbol{D}=\operatorname{diag}\left(D^{(1)}, \cdots, D^{(t)}\right)_{i \theta_{p_{s}}^{(s)}}$

where each $\boldsymbol{D}^{(s)}=\delta_{s} \operatorname{diag}\left(e^{i \theta_{1}^{(s)}}, \cdots, e^{i \theta_{p_{s}}}\right)$ with $\left|\delta_{1}\right|>$ $\left|\delta_{2}\right|>\cdots>\left|\delta_{t}\right|$. As a special case, if $D$ is locally primitive, then $D$ can be written as

$$
\boldsymbol{D}=\operatorname{diag}\left(\delta_{1} I_{p_{1}}, \cdots, \delta_{t} I_{p_{t}}\right) \text {. }
$$

Now let us define $q_{s}=\sum_{j=0}^{s} p_{j} \quad(s=1, \cdots t$, $\left.q_{0}=p_{0}=0\right)$ and $Q_{u}\left\langle q_{i-1}: q_{i}\right\rangle=\left\{\Omega_{u}=\left(\omega_{1}, \cdots, \omega_{u}\right) \mid q_{i-1}\right.$ $\left.\omega_{1}<\cdots<\omega_{u}<q_{i}\right\}$. Next, suppose $A_{N}=\left[a_{i j}^{(N)}\right]_{n \times r}$ is a sequence of $n \times r$ matrices and let

$$
A_{N}=Y_{N} G_{N}=\left[y_{i j}^{(N)}\right]_{n \times r}\left[g_{i j}^{(N)}\right]_{r \times r}
$$

be their GS factorization. Suppose $B$ is a $n \times r$ matrix, we can partition $B$ conformally as $D$ in (26). It is easily verified that the $(u, v)$ element of $(i, j)$ block $B_{i j}$ of $B$ is exactly the $\left(q_{i-1}+u, q_{j-1}+v\right)$ element of the whole matrix $B . B$ is said to satisfy condition $(\beta)$ if for each $k=q_{i-1}+u$ there exists $\Omega_{u} \in Q_{u}\left\langle q_{i-1}: q_{i}\right\rangle$ such that

$$
\operatorname{det} B_{\langle k\rangle}^{q_{i-1} \Omega u} \neq 0 .
$$

We now have the following theorem.

Theorem 4.2. Let $A_{N}$ be a sequence of $n \times r$ matrices of full column rank with GS factor $A_{N}=Y_{N} G_{N}$. Also suppose $D$ is a diagonal matrix and $D_{r}$ is $r \times r$ leading submatrix of $D$. Then

1) $D^{-N} A_{N}$ converges to $\widetilde{B}$ which satisfies condition $(\beta) \Leftrightarrow G_{N}$ converges and $D^{-N} Y_{N}$ converges to $Z$ which satisfies condition $(\beta)$

2) If in addition $D$ is decreasing, i.e. $D$ satisfies (26), then for $k=q_{i-1}+u$ and $l=q_{j-1}+v \quad(i \leq j-1)$

$$
\frac{y_{k l}^{(N)}}{d_{k}^{N}}=O\left(\left|\frac{\delta_{j}}{\delta_{i}}\right|^{2 N}\right) \text {. }
$$

Proof. 1) The sufficiency is obvious. So let us turn to the necessary part. For $\boldsymbol{D}=\operatorname{diag}\left(d_{1}, \ldots, d_{n}\right)$, there exists a permutation $Q$ such that $\hat{D}=Q^{*} D Q$ is decreasing. Meanwhile, by hypothesis and the fact that $D^{-N} A_{N}=Q$ 
$\left(Q^{*} D^{-N} Q\right)\left(Q^{*} A_{N}\right)=Q \hat{D}^{-N} \hat{A}_{N} \quad$ with $\quad \hat{A}_{N}=Q^{*} A_{N}$, it follows that $\hat{D}^{-N} \hat{A}_{N}$ converges. So without loss of generality, we assume that $D$ is decreasing and partition $D$ as (26) and simply consider $D^{-N} A_{N}$. We shall now, without risk of confusion, abbreviate the set $Q_{u}\left\langle q_{i-1}: q_{i}\right\rangle=\left\{\Omega_{u}=\left(\omega_{1}, \cdots, \omega_{u}\right) \mid q_{i-1}<\omega_{1}<\cdots<\omega_{u}<q_{i}\right\}$ to $Q_{u}$ and for $I=\left(i_{1}, \ldots, i_{s}\right)$ set $\pi_{I}=d_{i_{1}} \cdots d_{i_{s}}$. It at once follows that

$$
\left|\pi_{\left\langle q_{i-1}\right\rangle \Omega_{u}}\right|=\left|\pi_{\langle k\rangle}\right| .
$$

We now have from (23)

$$
\begin{aligned}
& g_{k l}^{(N)}=\operatorname{det}\left(A_{N}^{*} A_{N}\right)_{\langle k-1\rangle(I)}^{\langle k\rangle} / V_{k}^{2} \\
& =\frac{\sum_{I \in Q_{k, n}} \overline{\operatorname{det}\left(A_{N}\right)_{\langle k\rangle}^{I}} \cdot \operatorname{det}\left(A_{N}\right)_{\langle k-1\rangle(l)}^{I}}{\sum_{I \in Q_{k, n}}\left|\operatorname{det}\left(\left(A_{N}\right)_{\langle k\rangle}^{I}\right)\right|^{2}} \text { (from Cauchy-Binet) } \\
& =\frac{\left(\sum_{I \in\left\langle q_{i-1}\right\rangle Q_{u}}+\sum_{I \notin\left\langle q_{i-1}\right\rangle Q_{u}}\right) \overline{\operatorname{det}\left(A_{N}\right)_{\langle k\rangle}^{I}} \cdot \operatorname{det}\left(A_{N}\right)_{\langle k-1\rangle(I)}^{I}}{\left.\left(\sum_{I \in\left\langle q_{i-1}\right\rangle Q_{u}}+\sum_{I \notin\left\langle q_{i-1}\right\rangle Q_{u}}\right) \mid \operatorname{det}\left(A_{N}\right)_{\langle k\rangle}^{I}\right)\left.\right|^{2}} \\
& =\frac{\sum_{\Omega_{u} \in Q_{u}} \overline{\operatorname{det}\left(A_{N}\right)_{\langle k\rangle}^{\left\langle q_{i-1}\right\rangle \Omega_{u}}} \cdot \operatorname{det}\left(A_{N}\right)_{\langle k-1\rangle(l)}^{\left\langle q_{i-1}\right\rangle \Omega_{u}}+\sum_{I \notin\left\langle q_{i-1}\right\rangle Q_{u}}}{\sum} \\
& \sum_{\Omega_{u} \in Q_{u}}\left|\operatorname{det}\left(\left(A_{N}\right)_{\langle k\rangle}^{\left\langle q_{i-1}\right\rangle \Omega_{u}}\right)\right|^{2}+\sum_{I \notin\left\langle q_{i-1}\right\rangle Q_{u}} \\
& =\frac{\sum_{\Omega_{u} \in Q_{u}} \overline{\left(\frac{\operatorname{det}\left(A_{N}\right)_{\langle k\rangle}^{\left\langle q_{i-1}\right\rangle \Omega_{u}}}{\left(\pi_{\langle k\rangle}\right)^{N}}\right)} \cdot \frac{\operatorname{det}\left(A_{N}\right)_{\langle k-1\rangle(l)}^{\left\langle q_{i-1}\right\rangle \Omega_{u}}}{\left(\pi_{\langle k\rangle}\right)^{N}}+o\left(\left|\frac{\delta_{i+1}}{\delta_{i}}\right|^{2 N}\right)}{\sum_{\Omega_{u} \in Q_{u}}\left|\frac{\operatorname{det}\left(A_{N}\right)_{\langle k\rangle}^{\left\langle q_{i-1}\right\rangle \Omega_{u}}}{\left(\pi_{\langle k\rangle}\right)^{N}}\right|^{2}+o\left(\left|\frac{\delta_{i+1}}{\delta_{i}}\right|^{2 N}\right)},
\end{aligned}
$$

$$
\begin{aligned}
& \frac{y_{k l}^{(N)}}{d_{k}^{N}}=\frac{1}{d_{k}^{N}} \cdot \frac{\sum_{I \in Q_{l-1, n}} \operatorname{det}\left(A_{N}\right)_{\langle l\rangle}^{I(k)} \cdot \overline{\operatorname{det}\left(A_{N}\right)_{\langle l-1\rangle}^{I}}}{V_{l-1}^{2}} \\
& =\frac{1}{d_{k}^{N}} \frac{\left(\sum_{I \in\left\{q_{j-1}|k\rangle Q_{v}\right.}+\sum_{I \notin\left\{q_{j-1}|k\rangle Q_{v}\right.}\right) \operatorname{det}\left(A_{N}\right)_{\langle l\rangle}^{I(k)} \cdot \overline{\operatorname{det}\left(A_{N}\right)_{\langle l-1\rangle}^{I}}}{\left(\sum_{I \in\left\langle q_{j-1}\right\rangle Q_{v-1}}+\sum_{I \notin\left\{q_{j-1}\right\rangle Q_{v-1}}\right)\left|\operatorname{det}\left(A_{N}\right)_{\langle k-1\rangle}^{I}\right|^{2}} \\
& =\frac{\sum_{\Omega_{v} \in Q_{v}} \operatorname{det}\left(A_{N}\right)_{\langle l\rangle}^{\left\langle q_{j-1} k k\right\rangle \Omega_{v}(k)} \overline{\operatorname{det}\left(A_{N}\right)_{\langle l-1\rangle}^{\left\langle q_{j-1} \mid k\right\rangle \Omega_{v}}}+\sum_{I \notin\left\langle q_{j-1} \mid k\right\rangle Q_{v}}}{d_{l}^{N}\left(\sum_{\Omega_{v-1} \in Q_{v-1}}\left|\operatorname{det}\left(\left(A_{N}\right)_{\langle l \mid-1\rangle}^{\left\langle q_{j-1}\right\rangle \Omega_{v-1}}\right)\right|^{2}+\sum_{I \notin\left\langle q_{j-1}\right\rangle Q_{v-1}}\right)} \\
& =\frac{1}{d_{k}^{N}} \cdot \frac{\sum_{\Omega_{v} \in Q_{v}} \frac{\operatorname{det}\left(A_{N}\right)_{\langle l\rangle}^{\left\langle q_{j-1} \mid k\right\rangle \Omega_{v}(k)}}{\left(\pi_{\langle l-1\rangle}\right)^{N}}\left(\frac{\operatorname{det}\left(A_{N}\right)_{\langle l-1\rangle}^{\left\langle q_{j-1} \mid k\right\rangle \Omega_{v}}}{\left(\pi_{\langle l-1\rangle}\right)^{N}}\right)+\sum_{I \notin\left\langle q_{j-1}\right\rangle Q_{v}}}{\sum_{\Omega_{v-1} \in Q_{v-1}}\left|\frac{\operatorname{det}\left(\left(A_{N}\right)_{\langle l-1\rangle}^{\left\langle q_{j-1}\right\rangle \Omega_{v-1}}\right)}{\left.\pi_{\langle l-1\rangle}\right)^{N}}\right|^{2}+\sum_{I \notin\left\{q_{j-1} Q_{V-1}\right.}}
\end{aligned}
$$

Since $D^{-N} A_{N}$ convergence, so does the submatrices $\left(D^{-N} A_{N}\right)_{I}^{\left\langle q_{i-1} \Omega_{u}\right.}$ and their determinant and hence

$$
\begin{aligned}
\frac{\operatorname{det}\left(A_{N}\right)_{I}^{\left\langle q_{i-1}\right\rangle \Omega_{u}}}{\left(\pi_{\left\langle q_{i-1}\right\rangle \Omega_{u}}\right)^{N}} & =\operatorname{det}\left[\left(D_{\left\langle q_{i-1}\right\rangle \Omega_{u}}^{\left\langle q_{i-1}\right\rangle \Omega_{u}}\right)^{-N}\left(A_{N}\right)_{I}^{\left\langle q_{i-1}\right\rangle \Omega_{u}}\right] \\
& =\operatorname{det}\left(D^{-N} A_{N}\right)_{I}^{\left\langle q_{i-1}\right\rangle \Omega_{u}}
\end{aligned}
$$

converges, say, to $\operatorname{det}\left(\tilde{A}_{N}\right)_{I}^{\left\langle q_{i-1}\right\rangle \Omega_{u}}$. We have that consequently (31) converges to

$$
\frac{\sum_{\Omega_{u} \in Q_{u}} \overline{\operatorname{det}\left(\tilde{A}_{\langle k\rangle}^{\left\langle q_{i-1}\right\rangle \Omega_{u}}\right) \cdot \operatorname{det}\left(\tilde{A}_{\langle k-1\rangle(l)}^{\left\langle q_{i-1}\right\rangle \Omega_{u}}\right)}}{\sum_{\Omega_{u} \in Q_{u}}\left|\operatorname{det}\left(\tilde{A}_{\langle k\rangle}^{\left\langle q_{i-1}\right\rangle \Omega_{u}}\right)\right|^{2}},
$$

in which the denominator is nonzero as $\widetilde{A}$ satisfies condition $(\beta)$. Hence $G_{N}$ converges and this implies that $D^{-N} Y_{N}=D^{-N} A_{N} G_{N}^{-1}$ also converges.

2) Lastly, what remains is to show that $Y_{N} D_{r}^{-N}$ converges if $D$ is decreasing, i.e. $D$ satisfies (26). Now for $k=q_{i-1}+u, l=q_{j-1}+v \quad(i \leq j-1)$, it follows that 


$$
\begin{aligned}
& =\frac{\left|d_{l}\right|^{2 N}}{\left|d_{k}\right|^{2 N}} \cdot \frac{\sum_{\Omega_{v} \in Q_{v}} \frac{\operatorname{det}\left(A_{N}\right)_{\langle l\rangle}^{\left\langle q_{j-1} \mid k\right\rangle \Omega_{v}(k)}}{\left(\pi_{\langle l\rangle}\right)^{N}} \overline{\left(\frac{\operatorname{det}\left(A_{N}\right)_{\langle l-1\rangle}^{\left\langle q_{j-1} \mid k\right\rangle \Omega_{v}}}{\left(\pi_{\langle l k\rangle}\right)^{N}}\right)}+o\left(\left(\frac{\delta_{j+1}}{\delta_{i+1}}\right)^{2 N}\right)}{\sum_{\Omega_{v-1} \in Q_{v-1}}\left|\frac{\operatorname{det}\left(\left(A_{N}\right)_{\langle l-1\rangle}^{\left\langle q_{j-1}\right\rangle \Omega_{v-1}}\right)}{\left(\pi_{\left\langle q_{j-1}\right\rangle \Omega_{v-1}}\right)^{N}}\right|^{2}+o\left(\left(\frac{\delta_{j+1}}{\delta_{j}}\right)^{2 N}\right)}
\end{aligned}
$$

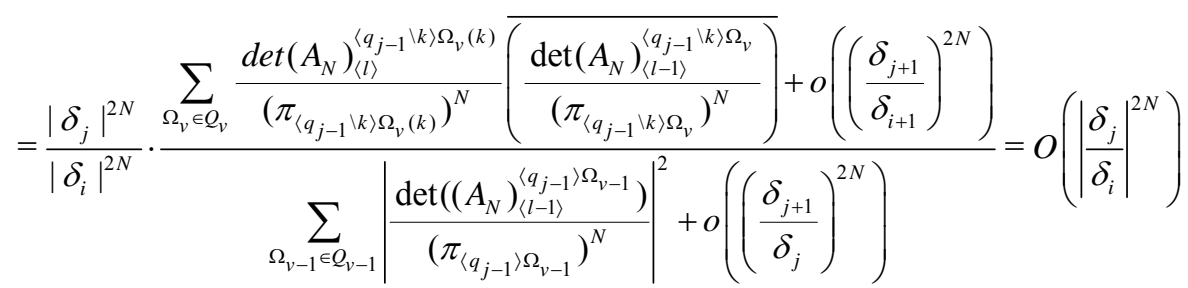

This completes the proof of 2).

As a consequence of the above theorem we have

Corollary 4.3. Suppose $D$ is decreasing and $A_{N}$ 's have orthogonal columns. If $D^{-N} A_{N}$ converges to $\widetilde{B}$ which satisfies condition $(\beta)$, then for $k=q_{i-1}+u$ and $l=q_{j-1}+v \quad(i \leq j-1)$

$$
\frac{a_{k l}^{(N)}}{d_{k}^{N}}=O\left(\left|\frac{\delta_{j}}{\delta_{i}}\right|^{2 N}\right)
$$

Proof. In this case the GS factorization of $A_{N}$ are $A_{N}=A_{N} I_{r}$. So the result is the direct consequnce of Theorem 4.2.

Lemma 4.4. Suppose $D$ is decreasing and $A_{N}$ 's are of full column rank. If $B_{N}=\left[B_{p q}^{(N)}\right]=D^{-N} A_{N}$ converges, say, to $\widetilde{B}$, then $A_{N} D_{r}^{-N}$ converges iff

1) $\left(\tilde{B}_{j j}\right)_{u, v}=\tilde{B}_{q_{j-1}+u, q_{j-1}+v} \neq 0 \Rightarrow \theta_{u}^{(j)}=\theta_{v}^{(j)} \quad(j=1$, $\cdots, t)$

2) If $i<j$, then

$$
\left(\frac{\delta_{i}}{\delta_{j}}\right)^{N} B_{q_{i-1}+u, q_{j-1}+v}^{(N)} e^{i N\left(\theta_{u}^{(i)}-\theta_{v}^{(j)}\right)}
$$

converges.

Proof. It is not difficult to see that the $\left(q_{i-1}+u, q_{j-1}\right.$ $+v$ ) element of $A_{N} D_{r}^{-N}$ is

$$
\begin{gathered}
\left(A_{N} D_{r}^{-N}\right)_{q_{i-1}+u, q_{j-1}+v} \\
=\left(\frac{\delta_{i}}{\delta_{j}}\right)^{N} B_{q_{i-1}+u, q_{j-1}+v}^{(N)} e^{i N\left(\theta_{u}^{(i)}-\theta_{v}^{(j)}\right)} .
\end{gathered}
$$

As $B_{q_{i-1}+u, q_{j-1}+v}^{(N)}$ converges and $\left|\frac{\delta_{i}}{\delta_{j}}\right|<1$ for $i>j$, it follows that (32) converges to zero in this case. Hence $A_{N} D_{r}^{-N}$ converges iff i) and ii) hold.

Suppose $B$ is an $n \times n$ matrix and correspondingly there are $n$ nodes $S_{1}, S_{2}, \cdots, S_{n}$. We say that $B$ is indecomposable if for every $i$ and $j$

$$
\text { either } S_{i} \rightarrow \rightarrow S_{j} \text { or } S_{j} \rightarrow \rightarrow S_{i} .
$$

Next we have

Theorem 4.5. Let $A_{N}$ be of full column rank and $A_{N}=Y_{N} G_{N}$ be its GS factorization. Suppose $D^{-N} A_{N}$ converges, say, to $\widetilde{B}$ which satisfies $(\beta)$. Then the following statement are true

1) If $Y_{N} D_{r}^{-N}$ converges to $\tilde{\boldsymbol{Z}}=\operatorname{diag}\left(\tilde{Z}_{1}, \ldots, \tilde{Z}_{\mathrm{s}}\right)$ in which each block $\widetilde{Z}_{i}(i=1, \cdots, s)$ is indecomposable, then $D^{(s)}=\delta_{s} I_{p_{s}}, \quad s=1, \cdots, t$

$2)$ If $\quad D^{(s)} \stackrel{p_{s}}{=} \delta_{s} I_{p_{s}}, \quad s=1, \cdots, t$, then $Y_{N} D_{r}^{-N}$ converges.

Proof. From Theorem 4.2., the convergence of $B_{N}=$ $D^{-N} A_{N}$ implies the convergence of $G_{N}$ and $D^{-N} Y_{N}$. Suppose $D^{-N} Y_{N} \rightarrow \widetilde{Z}$. Then it follows, on account of Lemma 4.4, that $Y_{N} D_{r}^{-N}$ converges to $\tilde{\mathbf{Z}}=\operatorname{diag}\left(\tilde{Z}_{1}, \cdots\right.$, $\left.\tilde{Z}_{s}\right)$ if
a) $\left(\tilde{Z}_{j}\right)_{u, v}=\tilde{Z}_{q_{j-1}+u, q_{j-1}+v} \neq 0 \Rightarrow \theta_{u}^{(j)}=\theta_{v}^{(j)}$, and
b) if $i<j$, then

$$
\begin{gathered}
\left(Y_{N} D_{r}^{-N}\right)_{q_{i-1}+u, q_{j-1}+v} \\
=\left(\frac{\delta_{i}}{\delta_{j}}\right)^{N}\left(D^{-N} Y_{N}\right)_{q_{i-1}+u, q_{j-1}+v} e^{i N\left(\theta_{u}^{(i)}-\theta_{v}^{(j)}\right)}
\end{gathered}
$$

converges to zero. Now Corollary 4 says that for $i<j$

$$
\left(D^{-N} Y_{N}\right)_{q_{i-1}+u, q_{j-1}+v}=\frac{Y_{q_{i-1}+u, q_{j-1}+v}^{(N)}}{d_{q_{i-1}+u}^{N}}=O\left(\left|\frac{\delta_{j}}{\delta_{i}}\right|^{2 N}\right)
$$

and so b) is automatically statisfied in this case. Therefore $Y_{N} D_{r}^{-N}$ converges iff a) holds. Since each $\widetilde{Z}_{i}$ is indecomposable, for arbitrary $(u, v)$ there exists a path either from $S_{q_{i-1}+u}$ to $S_{q_{i-1+v}}$ or vice verse. In either case this implies that $\theta_{u}^{(i)} \stackrel{q_{i-1}+v}{=} \theta_{v}^{(i)}$ for any $u$ and $v$. We complete the proof of 1 ).

2) This time $D$ is locally primitive, so we have $\theta_{u}^{(j)}=\theta_{v}^{(j)} \quad(j=1, \cdots, t)$ and hence 


$$
\begin{gathered}
\left(Y_{N} D_{r}^{-N}\right)_{q_{i-1}+u, q_{j-1}+v} \\
= \begin{cases}\left(\frac{\delta_{i}}{\delta_{j}}\right)^{N}\left(D^{-N} Y_{N}\right)_{q_{i-1}+u, q_{j-1}+v} e^{i N\left(\theta_{u}^{(i)}-\theta_{v}^{(j)}\right)} & \text { if } i \neq j \\
\left(D^{-N} Y_{N}\right)_{q_{j-1}+u, q_{j-1}+v} & \text { if } j=i\end{cases}
\end{gathered}
$$

By hypothesis, the above converges for $j=i$. The convergence for $i>j$ is obvious; while the convergence for $i<j$ can be easily achieved by noticing that

$$
\left(D^{-N} Y_{N}\right)_{q_{i-1}+u, q_{j-1}+v}=\frac{Y_{q_{i-1}+u, q_{j-1}+v}^{(N)}}{d_{q_{i-1}+u}^{N}}=O\left(\left|\frac{\delta_{j}}{\delta_{i}}\right|^{2 N}\right) .
$$

Remark. From Theorem 4.5. we know that in the case of multiple eigenvalues, if $k=q_{i-1}+u$, then

$$
\frac{y_{k}^{(N)}}{d_{i}^{N}} \rightarrow[\overbrace{0, \cdots, 0}^{1 \cdots q_{i-1}}, \quad \overbrace{\tilde{p}_{q_{i-1}+1}, \cdots, \tilde{p}_{q_{i}}}^{\left(q_{i-1}+1\right) \cdots q_{i}}, 0, \cdots, 0]^{T} .
$$

Let us now turn to the applications of this theorem. Our first application is the following result gives the general convergence result of power scaled triangular matrix.

Corollary 4.6. Let $D$ be diagonal and $T$ be upper triangular. Then $D^{-N} T^{N}$ converges if and only if

1) Either $\left|\lambda_{i} / d_{i}\right|<1$ or $\lambda_{i}=d_{i}$ for each $i$

2) If $S_{i} \rightarrow \rightarrow S_{j} \Rightarrow\left|\lambda_{i}\right|>\left|\lambda_{j}\right|$.

Proof. Let $A_{N}=T^{N}$. This time the GS factorization for $A_{N}=T^{N}$ becomes $D_{T}^{N}\left(D_{T}^{-N} T^{N}\right)$ and from Theorem 4.2., $D^{-N} T^{N}$ converges if and only if both $G_{N}=D_{T}^{-N} T^{N}$ and $D^{-N} D_{T}^{N}$ converge.

The convergence of $D^{-N} D_{T}^{N}$ is equivalent to 1 ); while the convergence of $G_{N}=D_{T}^{-N} T^{N}$, on account of Theorem 3.4., is exactly the same as the path condition 2).

A relevant application of Theorem 3.4. is to the question of subspace iteration. Armed with Theorem 3.4. we can get a sharper theoretical result than was previously given.

\section{Application to the Subspace Iteration}

Next, suppose $T$ is an block upper diagonal matrix of the form

$$
\boldsymbol{T}=\left[\begin{array}{cccc}
\lambda_{1} I_{p_{1}} & T_{12} & \cdots & T_{1 t} \\
0 & \lambda_{2} I_{p_{2}} & \cdots & T_{2 t} \\
\vdots & \ddots & \ddots & \vdots \\
0 & \cdots & 0 & \lambda_{t} I_{p_{t}}
\end{array}\right]
$$

where $\left|\lambda_{1}\right|>\left|\lambda_{2}\right|>\cdots>\left|\lambda_{t}\right|$. Let $\boldsymbol{D}_{T}=\operatorname{diag}(T)=\operatorname{diag}$ $\left(\lambda_{1} I_{p_{1}}, \cdots, \lambda_{t} I_{p_{t}}\right)$ and denote $D_{T_{r}}=\left(D_{T}\right)_{\langle r\rangle}^{\langle r\rangle}$. Then from
Theorem 3.4. it follows that $D_{T}^{-N} T^{N}$ converges.

Assume $B$ is $n \times r$ matrix of full column rank. Therefore $r \leq n=\sum_{i=1}^{t} p_{i}$ and without loss of generality we can write $r=\sum_{i=1}^{s} p_{i}+w$ for some $w \leq p_{s+1}$. Thus we can write $T_{r}=\operatorname{diag}\left(\lambda_{1} I_{p_{1}}, \cdots, \lambda_{s} I_{p_{s}}, \lambda_{s+1} I_{w}\right)$. We now have

Corollary 5.1. Let $T$ be $n \times n$ upper triangular matrix defined as in (33), and let $B$ be $n \times r$ matrix whose columns are linearly independent. If

$$
T^{N} B=Y_{N} G_{N}
$$

is its GS factorization, then the followings hold

1) $D_{T}^{-N} T^{N} B$ converges, say, to a limit $\tilde{A}$.

2) $Y_{N} D_{T_{r}}^{-N}$ converges to $\left[\begin{array}{l}P \\ 0\end{array}\right]$, where $P=\operatorname{diag}\left(P_{1}\right.$, $\left.\cdots, P_{s}, \tilde{P}\right)$ and each $P_{i} \quad(i=1, \cdots, s)$ is a $p_{i} \times p_{i}$ matrix and $\widetilde{P}$ is a $p_{s+1} \times w$ matrix.

Proof. The result follows by simply choosing $A_{N}=T^{N} B$ in Theorem 4.2.

Let us now turn to the question of subspace iteration for a restricted class of matrices. Suppose that

$$
A=V T V^{*}
$$

is $n \times n$ matrix, where $V$ is unitary and $T$ is as in (33). Then using the same $P_{i}$ as above we have

Corollary 5.2. Suppose that $A$ is an $n \times n$ matrix which satisfies (34). let $Y_{0}$ be an $n \times r$ matrix whose columns are linearly independent and $\left\{Y_{N}\right\}$ be sequence of matrices defined by the following factorization

$$
A^{N} Y_{0}=Y_{N} G_{N}
$$

Then

$$
Y_{N} D_{T_{r}}^{-N} \rightarrow\left[V_{1} P_{1}, \cdots, V_{s} P_{s}, V_{s+1} \tilde{P}\right] .
$$

Proof. Since

$$
A^{N} Y_{0}=Y_{N} G_{N}
$$

it follows that

$$
V T^{N}\left(V^{*} Y_{0}\right)=Y_{N} G_{N} .
$$

Partition $V=\left[V_{1}, \cdots, V_{t}\right]$ conformally to that of $T$ in (33) and set $B=V^{*} Y_{0}$, then

$$
T^{N} B=\left(V^{*} Y_{N}\right) G_{N} .
$$

It is easily seen that the columns of $V^{*} Y_{N}$ are orthogonal. Therefore (36) can be regarded as the GS factorization of $T^{N} B$. From Corollary 5.1., we have that for $V=\left[V_{1}, \cdots, V_{t}\right]$

$$
V^{*} Y_{N} D_{T_{r}}^{-N} \rightarrow\left[\begin{array}{l}
P \\
0
\end{array}\right]
$$

which is equivalent to 
$Y_{N} D_{T_{r}}^{-N} \rightarrow V\left[\begin{array}{c}P \\ 0\end{array}\right]=V_{r} P=\left[V_{1} P_{1}, \cdots, V_{s} P_{s}, V_{s+1} \tilde{P}\right]$

\section{References}

[1] F. L. Bauer, "Das Verfahren der Treppeniteration und verwandte Verfahren zur Lösung Algebraischer Eigenwertprobleme," Zeitschrift für Angewandte Mathe- matik und Physik, Vol. 8, No. 3, 1957, pp. 214-235. doi:10.1007/BF01600502

[2] A. Ben-Israel, "A Volume Associated with $m \times n$ Matrices," Linear Algebra and Its Applications, Vol. 167, No. 1, pp. 87-111, 1992. doi:10.1016/0024-3795(92)90340-G

[3] X. Chen and R. E. Hartwig, "On Simultaneous Iteration for Computing the Schur Vectors of Matrices," Proceedings of the 5th SIAM Conference on Applied Linear Algebra, Snowbird, June 13-19, 1994, pp. 290-294.

[4] X. Chen and R. E. Hartwig, "On the Convergence of Power Scaled Cesaro Sums," Linear Algebra and Its Applications, Vol. 267, pp. 335-358, 1997. doi:10.1016/S0024-3795(97)80056-0

[5] X. Chen and R. E. Hartwig, "The Semi-iterative Method Applied to the Hyperpower Iteration," Numerical Linear Algebra with Applications, Vol. 12, No. 9, pp. 895-910, 2005. doi:10.1002/nla.429

[6] X. Chen and R. E. Hartwig, "The Picard Iteration and Its Application," Linear and Multi-linear Algebra, Vol. 54, No. 5, 2006, pp. 329-341. doi:10.1080/03081080500209703

[7] R. A. Horn and C. R. Johnson, "Matrix Analysis," Cambridge University Press, Cambridge, 1985.
[8] R. A. Horn and C. R. Johnson, "Topics in Matrix Analysis," Cambridge University Press, Cambridge, 1991.

[9] A. S. Householder, "The Theory of Matrices in Numerical Analysis," Dover, New York, 1964.

[10] B. N. Parlett and W. G. Poole, Jr., "A Geometric Theory for the QR, LU, and Power Iterations," SIAM Journal on Numerical Analysis, Vol. 10, No. 2, 1973, pp. 389-412. doi: $10.1137 / 0710035$

[11] H. Rutishauser, "Simultaneous Iteration Method for Symmetric Matrices," Numerische Mathematik, Vol. 16, No. 3, 1970, pp. 205-223. doi:10.1007/BF02219773

[12] Y. Saad, "Numerical Methods for Large Eigenvalue Problems," Manchester University Press, Manchester, 1992.

[13] G. W. Stewart, "Methods of Simultaneous Iteration for Calculating Eigenvectors of Matrices" In: John J. H. Miller, Eds., Topics in Numerical Analysis II, Academic Press, New York, 1975, pp. 185-169.

[14] G. R. Wang, Y. Wei and S. Qiao, "Generalized Inverses: Theory and Computations," Science Press, Beijing/New York, 2004.

[15] D. S. Watkins, "Understanding the QR Algorithm," SIAM Review, Vol. 24, No. 4, 1982, pp. 427-440. doi: $10.1137 / 1024100$

[16] D. S. Watkins, "Some Perspectives on the Eigenvalue Problem," SIAM Review, Vol. 35, No. 3, 1993, pp. 430-470, doi:10.1137/1035090

[17] J. H. Wilkinson, "The Algebraic Eigenvalue Problem," Oxford University Press (Clarendon), London and New York, 1964. 